\title{
La recomposición de la triple vocación de la ciencia social en América Latina
}

\author{
Manuel Antonio Garretón M. \\ Universidad de Chile, Santiago, Chile. \\ Email: magarret@uchile.cl
}

\begin{abstract}
Resumen: ${ }^{1}$ Las ciencias sociales se caracterizaron por el manejo de la tensión entre la pretensión científica y la dimensión intelectual crítica, buscando un cierto diagnóstico-imagen de la sociedad que permitiera su transformación. Sus tres grandes vocaciones, la científica, la crítica y la profesional estuvieron indisolublemente ligadas. En este artículo se examina la disociación actual de estas dimensiones y se discuten tres desafíos, la transdisciplinariedad, el horizonte del concepto límite y la convergencia de las diversas voces de las ciencias sociales en América Latina.

Palabras clave: Ciencias Sociales, Dimensión crítica, Dimensión científica, Dimensión profesional. Conceptolímite,América Latina.
\end{abstract}

\section{Therestructuring ofthetriplevocation of socialscience in Latin America}

\begin{abstract}
Social sciences characterized for managingthe tension betweenscientific pretensionsand the intellectualcriticismdimension,searching for a certainimage-diagnosis of society that would allowits transformation. These three greatvocations: scientific, critical and professional,wereinextricably linked. In this article,the currentdissociation of these dimensionsis examined, andthree challenges:transdisciplinarity,the horizon of thelimitconcept andthe convergence ofthe diverse voices ofthe social sciences inLatin Americaare discussed.

Keywords: Social Sciences, Critical dimension, scientificdimension, professional dimension. Limitconcept,LatinAmerica.
\end{abstract}

\section{A recomposição da triplicevocação da ciência social na América Latina}

Resumo: As ciências sociais se caracterizaram por gerir a tensão entre a pretensão científica e a dimensão intelectual crítica, procurando certo diagnósticoimagem da sociedade que permitiesse sua transformação. Suas três grandes vocações, a científica, a crítica e a profissional estiveram indissociavelmente ligadas. Neste artigo, se examina a atual dissociação dessas dimensões e discutem-se três desafios, a transdisciplinaridade, o horizonte do conceito limite ea convergência das diversas vozes das ciências sociais na América Latina.

Palavras-chave: Ciências Sociais, dimensão crítica, dimensão científica, dimensão profissional,Conceito limite, América Latina.

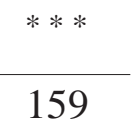


Las ciencias sociales se caracterizaron en nuestro continente por el manejo de la tensión entre la pretensión científica y la dimensión intelectual crítica, buscando un cierto diagnóstico-imagen de la sociedad que permitiera su transformación. Ello llevó a que las tres grandes vocaciones o "aguijones”, de la ciencia social como diría Medina Echavarría, la científica, la crítica y la profesional estuvieran indisolublemente ligadas. En este artículo examinaremos cómo se ha producido una disociación en la época actual de estas dimensiones y discutiremos algunos desafíos y tareas para restituir la capacidad a la vez de conocer, comprender y transformar con las herramientas que nos son propias.

\section{La disociación de las tres vocaciones de las ciencias sociales}

Partamos por una reflexión comparativa. A fines de la década de los 50 ' y mediados de los 60', las generaciones fundadoras y los primeros estudiantes de ciencias sociales, y hablaremos indistintamente de ciencias sociales o sociología, enfrentan un mundo definido que se quiere ayudar a cambiar. Se tenía relativamente claro, cualesquiera fuera la orientación teórica que luego se adoptara, tanto un cierto diagnóstico, una cierta critica a ese mundo social que podía llamarse sociedad subdesarrollada o capitalista dependiente., como una cierta teoría del cambio que podía llamarse modernización o desarrollo, o que podía llamarse revolución. Entonces, la tarea de la sociología era construir un tipo de conocimiento y profesionales que fueran capaces de realizar esa tarea. Dicho de otra manera, se sabía a qué mundo nos enfrentábamos y que mundo queríamos cambiar, pero no sabíamos qué éramos como científicos sociales. Había que inventar en este país y en el contexto latinoamericano, lo que eran la sociología y las ciencias sociales. Y lo mismo ocurrió en la época de la dictadura: hubo que reinventar una profesión para enfrentar un mundo con una meta clara.

En la actualidad, la condición de las ciencias sociales pareciese vivir un proceso diametralmente opuesto. La gran mayoría de los sociólogos y científicos sociales saben o conocen bien su oficio, y poseen gran destreza, a veces con cierta obsesión, en el uso de las técnicas y herramientas metodológicas para el análisis de problemas específicos, para escrutar la realidad, para hacer diagnósticos. Pero no sabemos en qué mundo estamos. Ni tampoco sabemos cómo se puede cambiar o qué mundo queremos. Las ciencias sociales tuvieron desde su fundación hasta hace poco, una o varias teorías sobre la sociedad y su cambio. El gran problema hoy parece ser precisamente la ausencia de una teoría de la sociedad o de su cambio. En palabras de uno de los grandes intelectuales latinoamericanos Carlos Monsiváis, "no entiendo lo que pasa y cuando lo entiendo ya pasó". Ese es el drama en cierto modo que viven nuestras disciplinas. Y esto tiene que ver con las transformaciones que han ocurrido en el mundo, pero también con problemas específicos de las disciplinas para comprenderlo. 
La pregunta que está en el aguijón intelectual y ético de la sociología y la ciencia social: ¿para qué? o ¿¿cuál es el tipo de sociedad que está detrás del problema que se estudia y que se quisiera superar?, presente en la génesis e institucionalización de las ciencias sociales, es hoy el componente más débil de su constitución. La vocación científica ha sido desprendida de la vocación intelectual, lo que en consecuencia permite obtener ciertos conocimientos parciales de lo social, pero no necesariamente comprenderlo. A su vez la vocación crítico intelectual desprendida de la científica y profesional, tiende a convertirse en una pura retórica testimonial. Del mismo modo, así como la vocación intelectual o crítica ha sido desprendida de la vocación científica, la dimensión profesional separada de la dimensión científica deviene en pura técnica instrumental, lo que se torna eficiente para realizar cosas, sin conocer su real "sentido mentado".

En este contexto tiende a producirse dos tipos de desviaciones respecto de lo que fue la vocación de la sociología. Por un lado, si bien las ciencias sociales han adquirido una práctica eficiente y diversificada del oficio profesional en las últimas décadas, ello ha estado muchas veces separado de la vocación científica e intelectual, lo que la ha remitido a un tipo de sociología con un alto componente tecnocrático. Podríamos hablar de unas ciencias sociales de los problemas y déficit en cada campo: diagnósticos del ámbito en cuestión, de las demandas de los sectores afectados, de los efectos subjetivos y objetivos de las políticas destinadas a resolver los problemas. En todo esto hay que reconocer una gran contribución de las ciencias sociales y en ningún caso ello debiera abandonarse. Pero cabe preguntarse si es posible otra perspectiva que vaya más allá de problemas y soluciones el sentido de las acciones sociales y políticas, es decir, que se interrogue por cuál es el tipo de sociedad que se quiere, que es la pregunta ético normativa y también teórica, que caracterizó la tensión constituyente entre ciencia y normatividad que atravesó a toda la teoría social desde su génesis hasta gran parte del siglo pasado (Mascareño, 2009).

Por otro lado, las insuficiencias de esta sin duda exitosa profesionalización de la ciencia social, ha llevado a una postura que reivindica solo la dimensión crítica y profética de la ciencia social y desconfía de todo conocimiento provisto por las metodologías y técnicas de que disponen. Al contrario de la sociología de los diagnósticos de problemas parciales, esta apunta sólo a la denuncia de un principio de totalidad dominante en la sociedad, donde el neoliberalismo aparece como la única explicación de todos los males y condena todo el proceso de formación profesional de los sociólogos porque éste lleva a la complicidad de los científicos sociales con el sistema. Frente al optimismo de la ilusión tecnocrática que al resolver los problemas deja de plantearse la cuestión del sentido de la acción social y la pregunta ética por el tipo de sociedad, aquí estamos ante una suerte de negatividad que retrata una sociedad imposible de transformar. Frente a una sociología que hace de la demanda aparente y del discurso de los actores, medidos cualitativa o cuantitativamente, su objeto acrítico de estudio, aquí estamos frente a la denuncia de actores totalmente dominados y cooptados por el sistema a los que hay que hay que iluminar sobre su condición. 
Entre estos dos extremos, la pregunta necesaria por el tipo de sociedad que se quiere conlleva otro problema que tiene que ver con la relación con los actores y sujetos. ¿Con qué autoridad pueden las ciencias sociales criticar radical o globalmente una sociedad o actores, por ejemplo consumistas, aspiracionales, adictos a los malls y reality shows, en que la mayoría reclama la extensión de consumo alienador o destructivo del medio ambiente? ¿Deben o no estas disciplinas expresar sólo los problemas que siente la "gente" y sus demandas o deben también someterla a la crítica desde la perspectiva de otro tipo de sociedad y ello en nombre de qué principio ajeno a la vida cotidiana de esa gente que se impone autoritativamente?

Así, la problemática nueva de la sociedad en nuestra región, reconstruir la polis refundando las relaciones entre Estado y sociedad a partir del principio de igualdad y reinsertarse en el mundo globalizado como bloque y modelo particular de modernidad (Garretón, 2012), requiere una ciencia social que piense y reflexione cómo se construye la sociedad y cómo se construyen sujetos individuales y colectivos que sean capaces de manejar las transformaciones del mundo, pero al mismo tiempo que sea capaz de refundar las relaciones entre Estado y sociedad, su orden económico y su orden político.

Ante la pregunta, cuál es la responsabilidad fundamental de las ciencias sociales, en pocas palabras es devolverse a sí misma esa unidad siempre llena de tensiones entre vocación científica, intelectual y profesional, superando a. las ilusiones tecnocráticas y proféticas, pero sin renunciar a la rigurosidad tanto científica como ético-crítica, en un mundo en que ellas hoy día son necesarias pero sus conocimientos son insuficientes para dar sentido a la vida de una sociedad. tarea.

En lo que sigue examinaremos tres cuestiones que atraviesan esta

\section{Disciplinas y transdiciplinariedad}

Una de las cuestiones principales planteadas a las ciencias sociales y a la sociología, tanto en América Latina como en otros contextos, es hasta qué punto puede cada disciplina definir el tipo de sociedad que se estudia y que se quiere. En el plano de la formación de científicos sociales se trata de seleccionar cuáles son los aportes que realizan las diversas disciplinas a la comprensión de la sociedad Ello precisamente con el fin explícito de entregar herramientas o formas de ver el mundo que le permitan al cientista social no quedar relegado a su dimensión exclusivamente profesional, que como señalamos, permite a los individuos poder obtener ganarse la vida, pero no necesariamente le da a su profesión otro sentido de trascendencia o pertenencia a una comunidad.

Lo cierto es que hoy día los enfoques, temáticas y abordajes, así como las técnicas de investigación caminan en sentido inverso a la especi- 
ficidad disciplinaria y cuesta distinguirlas en la práctica de trabajo. Hoy existen fenómenos complejos como la mundialización, la imposición de las lógicas del capital financiero transnacional, la irrupción de nuevos modelos de modernidad o la redefinición de las relaciones entre lo micro y macrosocial (Archer, 2009) a través de los procesos de constitución de las identidades colectivas, que redefinen el campo disciplinario. Por otro lado, cada una de las ciencias sociales, con la excepción de la economía fueron débiles en tanto ingenierías sociales es decir, en su capacidad de intervención, más allá de su capacidad de análisis u comprensión ${ }^{[2]}$ y hoy desde diversos campos aparecen ciertas técnicas, ciertas ingenierías sociales con debilidad de conocimientos sobre lo social, pero con gran capacidad de marketing y de resolver problemas concretos, que obligan a las ciencias sociales al menos dar cuenta de ellas, sino de incorporarlas en el proceso de formación. Disciplinas nuevas como la comunicación, el urbanismo, la ecología, por nombrar solo algunas, invaden el campo antes monopolizado por las ciencias sociales. De este modo, pareciera estar cambiando, bajo el florecimiento de disciplinas variadas, el paradigma de las ciencias sociales que se basó en la univocidad de un determinado objeto para cada disciplina. Aparece así como inevitable una fase "plástica", que puede durar mucho tiempo, en que coexiste la diferenciación formal en términos de carreras y formación y la semejanza sustantiva de los trabajos de todas estas disciplinas y de las nuevas especialidades que surgen. Ello no es muy distinto a lo señalado por el Informe Gubelkian (Comisión Gubelkian, 1996) y la necesidad de "abrir las ciencias sociales", obligando a pensar en nuevos paradigmas disciplinarios, donde los fenómenos de las sociedades a los que estamos asistiendo sean replanteados combinando las formas de trabajo más clásicas con las emergentes que apelan a la interdisciplinariedad o, más allá, a la transdiciplinariedad.

Esta última aparece como un factor importante a en pos de recuperar la pretensión intelectual y crítica de las ciencias sociales modernas. En tal sentido, como ya señalamos, las ciencias sociales y particularmente la sociología, están atravesadas por la tensión entre su pretensión científica y sus componentes normativos y de comprensión más allá de la ciencia estrechamente definida (Archer, 2009; Mascareño, 2009). Pero habría que distinguir la transdisciplinariedad de aquellas visiones que descartan radicalmente las disciplinas en pos de una única ciencia social, olvidando que la formación disciplinaria aparece hoy como insustituible para la iniciación en el mundo de lo científico social, que muchos problemas sí pueden resolverse desde una disciplina y que de las disciplinas provienen hasta hoy los marcos teóricos y muchos de los conocimientos empíricos que se requieren para la comprensión de la sociedad.

La transdisciplinariedad supone un conjunto de disciplinas que tratan de explicar el comportamiento individual y colectivo. En esa complejidad, existen disciplinas que no necesariamente tienen un carácter científico estricto, como hay otras que poseen dicha naturaleza. Las humanidades, la literatura y el cine proveen también conocimientos que escapan a lo estrechamente científico y que deben ser considerado en un enfoque 
transdiciplinario del estudio de la sociedad de la sociedad y en la formación de los profesionales en ciencias sociales. Por otro lado, hay temáticas que corresponden a teorías científico sociales, como el género, el territorio, las identidades, la memoria (que tiene hoy un componente biológico fundamental) que no pueden ser comprendidas solo desde una disciplina ni siquiera del conjunto de las disciplinas de las ciencias sociales y obligan a una visión no solo transdiciplinaria de las ciencias sociales sino con los otros mundos científicos. Pero ello a su vez, exige el permanente desarrollo de cada una de éstas y no su desaparición o fusión.

Un punto importante de la transdisciplinareidad en las ciencias sociales es la necesidad de la incorporación de la historia y la economía. La actual disociación que aqueja la condición de las disciplinas refleja no sólo la incapacidad de comunicación entre las disciplinas, sino que expresa una errónea comprensión de los fenómenos que se pretende analizar. En tal sentido, hay que abrir las ciencias sociales en la misma dirección que planteaba Wallerstein, si no, se sigue cayendo en un sesgo no sólo científico, sino también ideológico. Ejemplo de este sesgo lo da el tratamiento de la pobreza como categoría económica o estadística, desconociendo sus otras dimensiones.

Pero reconozcamos que todos estos temas tienen como trasfondo el cambio profundo de la sociedad contemporánea, muy distinta a la sociedad industrial de Estado nacional, de la que provienen las ciencias sociales tal cual las conocemos. Un nuevo tipo societal y nuevas formas de relaciones y acción sociales como las que se viven hoy deberán llevar a redefiniciones muy profundas tano de la dimensión profesional y científica como de la dimensión intelectual.

\section{La cuestión teórica. ¿De qué sociedad se trata?}

Como expresamos al inicio, cuando nacen las ciencias sociales en nuestro contexto tienen como intencionalidad comprender y tratar de transformar un determinado mundo que se puede delinear y definir gracias a un conjunto de teorías y enfoques que permiten avizorar qué es ese mundo que se tiene al frente y que nos modela. En tal sentido, las ciencias sociales se abocan al análisis de las sociedades del mundo transformándola en un objeto de estudio, pero además, se ven orientadas bajo un concepto normativo que apunta al horizonte deseable al cual se pretende avanzar. Este concepto, explícita o implícitamente, permitió organizar las prácticas profesionales y académicas en función de un sentido, que denominaremos como “concepto límite”. El concepto límite es un objeto de estudio sociológico, pero también es la perspectiva o si se prefiere, el horizonte utópico. ${ }^{3}$

En el origen de las ciencias sociales en América Latina este concepto fue el "desarrollo", que las ciencias sociales no económicas llamaron "modernización”. Ello dio origen a una serie de teorías y estudios que tenían que ver precisamente con este fenómeno que resolvía, o que intentaba 
resolver, uno de los grandes conflictos que tenía el mundo basado en la división norte-sur o entre países ricos y pobres.

El desarrollo era entonces el proceso que había que estudiar pero también estimular, atendiendo al descubrimiento de sus mecanismos de funcionamiento, con el fin de describir y explicar, pero también apoyar a los distintos actores sociales para llegar a tal estado de progreso. Este concepto límite comienza a entrar en crisis en los países subdesarrollados, una vez que emerge un nuevo tipo de confrontación en los países más avanzados, a saber, del mundo que estaba dividido bajo los conceptos de capitalismo / socialismo, dicotomía clásica de la guerra fría. El concepto límite que va a reemplazar al concepto de desarrollo, se llama “revolución”, asociado a la transformación del tipo de sociedad capitalista en una de tipo socialista.

Este desplazamiento del concepto límite, ahora llamado revolución, también organizó la práctica de los científicos sociales, donde hubo diversas teorías, métodos de investigación, así como formas de intervención social. De tal forma, esta nueva ciencia adquiría un compromiso más directo con los actores involucrados en el cambio social, haciendo más explícitos su componente normativo con la transformación de la sociedad, de la mano de quienes eran definidos como sus principales protagonistas.

La derrota o el fracaso de esos proyectos de sociedad - y los términos derrota o fracaso no son indiferentes, por cuanto el primero implica solo denuncia de un hecho y el segundo revisión de las categorías con que fueron pensados los proyectos- que conllevaban modelos o proyectos de ciencias sociales, dio origen a las dictaduras militares que se instalaron en los sesenta y setenta principalmente en el Cono Sur. Las ciencias sociales ensayaron distintas denominaciones para referirse a este fenómeno, con conceptos tales como dictaduras fascistas, fascistas dependientes, dictaduras de seguridad nacional, neo autoritarismos, Estados burocrático- autoritarios, entre otros. Precisamente, ante el fenómeno sociopolítico del autoritarismo que aquejaba a la sociedad, y junto con ello, a los proyectos emancipadores latentes en las ciencias sociales, se produjo un nuevo desplazamiento del concepto límite, estructurándose en torno al concepto de “derechos humanos” y, posteriormente, en torno al concepto límite “democracia”. Bajo este concepto', crítico de la situación imperante, se ensayaron distintas orientaciones teóricas. La novedad es que por primera vez se estaba ante un concepto límite que abarcaba no la totalidad de las dimensiones de la sociedad sino el régimen político. Ello, finalmente, reestructuró las ciencias sociales ya no basadas en grandes paradigmas de interpretación, sino más bien en la utilización de "teorías de alcance medio" para referirse al fenómeno de la transición, la democratización o el término de las dictaduras.

A pesar de que los conceptos límites fueron esenciales como organizadoras, latentes o manifiestos, de la práctica de los científicos sociales y como horizonte normativo, una lectura crítica de ellos también debe indicar que sufrieron una suerte de naturalización, en los cuales no se discutieron 
sus significados, una especie de chip que se importa sin desentrañar su contenido y circuitos internos, y que se reemplazan por otros que pueden ser alternativos pero que se les pensó de la misma manera, desde una perspectiva teleológica: se trata de llegar a esa sociedad meta. En tal sentido, cuando las ciencias sociales se referían al desarrollo, no se discutía teóricamente lo que ésta significaba sino que se analizaba el proceso que lleva a ella De igual forma, cuando las ciencias sociales discutían sobre modernización, no se planteaba el tema de la modernidad. Ello también ocurrió cuando se hablaba de socialismo o de cambio en el sistema capitalista, donde no se discutía mayormente la sociedad socialista. Se suponía que ahí estaban esas sociedades a las que se aspiraba y de lo que se trataba era llegar a ella. Esta tendencia, también se repitió para el concepto límite de democracia, sobre lo cual se tenía una cierta idea de lo que no era democrático, que estaba dado fundamentalmente por el carácter autoritario de la dictadura. Y lo que era democracia era fundamentalmente lo que existía en otras sociedades, por lo tanto, si bien los conceptos limites sirvieron para darle el sentido y llenar de contenido prácticas y conocimientos, temáticas de investigación, teorizaciones, ellos no fueron recreados o reinventados, sino que más bien quedaron entrampados en su origen teórico o de referente histórico.

Sin entrar en una discusión sobre la necesidad o no para el desarrollo de las ciencias sociales de un concepto límite como lo hemos señalado, lo que puede ser discutible en otros contextos histórico culturales, no puede negarse la trascendencia que ha tenido en el desarrollo de las ciencias sociales en América Latina, precisamente porque daba un sentido a las disciplinas y las ligaba de algún modo a procesos socio-históricos a los que querían contribuir, es decir, se imputaba un significado a la acción colectiva y nos acercábamos a los sujetos y actores. También es cierto que en esta vinculación con procesos y actores sociales reside una causa importante de su ideologización en ciertos períodos. Pero su ausencia expresa una cierta perplejidad frente al mundo que se estudia.

Y esto último está relacionado con algo que hemos planteado más arriba y es la dificultad de definir el tipo de sociedad en que se vive y la ausencia de una teoría de la sociedad y de su cambio. En otras épocas, la sociedad aparecía organizada, según la teoría que se tuviera, en torno a la economía, al Estado, la cultura, las clases u organizaciones sociales como la familia, la política. Hoy día, y eso se refleja en las denominaciones de sociedad posmoderna, sociedad líquida, sociedad red, no estamos en presencia de ejes vertebradores de la sociedad de estados nacionales. Se nos hace entonces muy difícil entender de qué se trata. Cuál es la problemática central de estas sociedades, .lo que llamamos el mundo de hoy, se nos aparece como algo mucho menos claro. Porque básicamente lo que ha ocurrido es que el fenómeno central al cual aplicábamos estos conceptos limites era el de la sociedad de Estado nacional ${ }^{4}$. Y el concepto de Estado nacional hoy día aparece fundamentalmente resquebrajado, desarticulado. Estamos frente a sociedades en las cuales tenemos un conjunto de procesos cuyo sentido no entendemos bien, sociedades fragmentadas que nos permiten definirla como des-igualitaria o desigual, pero sin claridad sobre la 
sociedad alternativa. Muchas de las respuestas sobre la naturaleza de las sociedades contemporáneas no hacen posible una ciencia social que responda a su fundamento central cual es comprender y transformar las sociedades. Por ejemplo, si éstas se nos aparecen como reducida a individuos o sujetos personales solamente, ella no puede cambiarse. Si no se entiende el mundo como algo distinto a la suma de individuos y proyectos personales o de grupos particulares, ni éste interesa ni puede cambiarse. Lo que puede cambiarse es uno mismo, al límite el cuerpo (pensemos en auge de tatuajes, piercings, cirugías plásticas, etc), o el entorno inmediato y las relaciones con los otros, al límite se redefine el concepto mismo de amistad que siempre implicó relaciones interpersonales directas con lazos de sentido afectivo y que hoy es aquel a quien no se conoce y se sale a buscar en el mercado virtual. Si me refiero a este ejemplo puntual es porque ilustra muy bien que las relaciones sociales o la acción social (concepto básico de las ciencias sociales) actuales, parecieran prescindir de eso que era lo central de los últimos doscientos años: una comunidad política, una relación entre Estado y la gente, en la que el trabajo cumplía un papel central en ella. Debilitamiento del concepto de trabajo y de política, por ejemplo, implican en el fondo un golpe muy fuerte a la sociología y a las ciencias sociales que nacieron para entender básicamente la sociedad moderna y sus transformaciones, es decir, una sociedad de Estado y una sociedad industrial y de trabajo. Entonces y con esto volvemos a lo señalado más arriba, tenemos instrumentos, tenemos metodologías, tenemos cuerpos teóricos, pero no entendemos el mundo y la sociedad en que vivimos. Y frente al malestar ante la desigualdad y el abuso, las respuestas tienden a ser o el conocimiento experto para resolver problemas y déficits a través de políticas públicas o preguntarse qué quieren los individuos, qué quiere la gente a través de encuestas o grupos de discusión.

Así, la idea de un proceso o un eje vertebrador parece haber perdido sentido. Estamos en sociedades frente a las cuales no tenemos una teoría del cambio porque no tenemos una teoría clara sobre lo que son. Porque una de las cosas fundamentales que han significado los procesos de desvertebración de la sociedad a través del debilitamiento de los Estados nacionales, de los procesos de explosión de identidades, de la globalización, es que ha perdido importancia, relevancia o sentido, aquella actividad o dimensión que articulaba y daba sentido al conjunto de la sociedad, que hacía que ella fuera algo más que un agregado de individuos en un territorio sin proyecto colectivo. En el caso latinoamericano ello era la política, a diferencia de otras sociedades donde pudo haber sido la economía o de otras donde pudo haber sido la cultura o la religión. En las sociedades nuestras la política fue el elemento fundante. No solo de la sociedad y su proyecto sino también de una relación particular con las subjetividades, con las trayectorias biográficas. Lo que tenemos hoy día es un estallido de este cemento de la sociedad.

Por ello, hemos indicado en diversos trabajos (Garretón, 2012), que independientemente de los modelos particulares que tenga cada sociedad concreta, sí hay una problemática central que tienen todas las sociedades 
latinoamericanas, es la reconstrucción de la polis, la construcción de las relaciones entre Estado y sociedad, más allá del desarrollo, la revolución o la democracia.

Ante esta situación cabe volver a plantear la certeza básica en que se fundan las ciencias sociales, que más allá de ser teórica, tiene también un componente ético: No es posible explicar ningún proceso o fenómeno individual o respecto de la subjetividad de los individuos, sin referirse a la interpretación de la sociedad y todo lo que ocurre en las sociedades tiene directa implicancia en la vida y trayectoria de los individuos. La premisa ontológica que asume este enunciado es que los cambios a nivel de la estructura son percibidos por los seres humanos, son vivenciados por los agentes que componen la sociedad (Archer, 2009). Esto es esencial de no perder de vista, en una sociedad en que los medios, los grupos corporativos, los poderes fácticos y muchas veces las políticas de gobierno tienden a negar la existencia de la sociedad: sólo bastarían los individuos y quienes pueden interpretarlos a nivel de los medios de comunicación, las encuestas o la tecnocracia.. La herencia del liberalismo se hace carne en este tipo de expresiones, donde todo recae en las personas o individuos o grupos particulares, restándole importancia a la sociedad y la solidaridad social que permite la existencia de aquéllos Pero hay otros que nos dicen: no son necesarios los individuos y las subjetividades, basta con los movimientos sociales o las sociedades globalizadas y sus poderes facticos. Entonces desde el punto de vista teórico la gran tarea es la reconstitución de este lazo entre personas y sociedad, lo que Martuccelli expresaba como individualizar los problemas societales y socializar los problemas individuales. O podríamos decirlo en términos más de sociología y ciencia políticas: hay que ciudadanizar la política y hay que politizar la ciudadanía. Somos productos sociales, pero también tenemos injerencia en la transformación de la sociedad a partir de las acciones que realizamos. Es tarea necesaria de las ciencias sociales asumir ese principio y defenderlo con fuerza ante la amenaza que ejercen los poderes que desde el sentido común y los criterios de la rentabilidad, niegan importancia al trabajo científico de las ciencias sociales en su tarea de explicar los funcionamientos de la sociedad que vivimos, y cómo transformarla.

\section{Universalidad científica o comprensión de “otra” realidad}

Las ciencias sociales en América Latina han sido expresión de una región golpeada por invasiones, dictaduras y sucesos que han afectado directamente a sus sociedades. Así, en el pensamiento social latinoamericano, siempre hubo permanente tensión entre el principio de universalidad de las ciencias, característica esencial de las ciencias sociales modernas, y la particularidad de los procesos sociales y políticos propios de las sociedades latinoamericanas. Ello ha sido una característica esencial de las ciencias sociales en la región, de la cual Chile no puede abstraerse: el desarrollo de ellas, desde los inicios hasta la actualidad, ha sido permeado por los procesos estructurales y culturales de la sociedad. El papel crítico e intelectual 
que han desarrollado las ciencias sociales en la región y en nuestro país no ha rehusado a su condición científica. Ello distingue a la ciencia social en este contexto de la desarrollada en países cuyas comunidades científicas se orientan más por los principios de la neutralidad que por objetivos normativos.

Desde su origen las ciencias sociales en este contexto han estado atravesadas por dos grandes visiones de carácter contradictorio que se hicieron evidentes el último tiempo a propósito de los bicentenarios de la independencia. En efecto, se ha asistido a celebraciones y conmemoraciones contradictorias entre quienes ven en la historia de la post independencia, la afirmación de identidades y voluntades nacionales, y quienes en cambio, sienten trágicamente que "no hay nada que celebrar" por cuanto tal historia ha sido la de la dominación e imposición de voluntades por parte de potencias externas y poderes domésticos (Monsiváis, 2001).

En el mismo nacimiento de las ciencias sociales como disciplinas académicas en la región primó una preocupación por la sociedad latinoamericana, por encima de una la búsqueda de teorías generales de la sociedad y del comportamiento colectivo. De algún modo ellas nacen, especialmente la sociología que fue la disciplina predominante por un par de décadas, como sociología del cambio social, donde el desarrollo o la modernización eran las formas específicas del cambio social en América Latina (Medina Echavarría, 1964; Faletto y Cardoso, 1968). La respuesta a los enfoques estructural funcionalistas de esta problemática, expresada teóricamente en el marxismo y, analíticamente, en el enfoque de la dependencia, es una variante crítica de una visión que pone el énfasis en los procesos estructurales y políticos, que analiza avances y fracasos, que realiza balances, en algunos casos más positivos, en otro negativos, de lo que han o no han llegado a ser nuestras sociedades ${ }^{5}$. Por ello, las ciencias sociales en América Latina han tenido, a pesar de su fuerte pretensión de cientificidad, una tensión constituyente con un componente normativo, que ha orientado la acción social hacia la transformación social, en sus más diversas variantes.

Los grandes déficits en la construcción de estas sociedades, en cuanto comunidades socio-económicas, políticas y culturales, están detrás de otra visión que tiene una expresión más antropológica y cultural que sociológica o política. Quizás la mejor síntesis del punto de partida de esta otra visión, sea el que la nación étnica fue avasallada por la nación cívica y que la realidad colonial no ha sido nunca superada por estas sociedades (Stavenhagen, 2000) El énfasis aquí no estará en el análisis de estructuras y procesos, sino en una visión de las fragmentaciones, los desgarros y la búsqueda de sujetos que expresan dimensiones de género, territoriales, étnicas, culturales, de sociedad civil, siempre oprimidas por hombres, blancos, metrópolis, militares o políticas institucionalizadas, es decir, más cercana a la problemática de las modernidades múltiples que de los modelos de modernización (García Canclini, 1980). No hay aquí balances frustrados u optimistas sino rechazo a la historia que conmemoraron tanto el V Centenario de la Conquista española 1992 como los bicentenarios de las indepen- 
dencias de España Los trabajos en torno a la colonialidad, el eurocentrismo, la constitución de sujetos opuestos a la racionalidad occidental, son expresiones de esta visión. ${ }^{6}$

Pareciera ser que estamos hoy en una situación en que es posible la convergencia de estas voces de las ciencias sociales, las que pueden basarse o llevar a posiciones políticas de izquierda y derecha, en la nueva problemática de Latinoamérica, que consiste en la refundación de las relaciones entre Estado y sociedad a partir del principio de igualdad para conformar un nuevo modelo de modernidad en el mundo globalizado. 


\section{Notas}

${ }^{1}$ Hemos usado en este artículo material de nuestro libro reciente Las ciencias sociales en la trama de Chile. Transformaciones socio-políticas y movimiento social. LOM (2014).

${ }^{2}$ He tratado estos temas en Garretón (2000), capítulo 1.

${ }^{3}$ Hemos adoptado libremente (Garretón 2007) la idea de concepto límite de Franz Hinckelammert (1984).

${ }^{4}$ Ello ha llevado a Touraine (2013) a hablar del fin de las sociedades.

${ }^{5}$ Una síntesis del desarrollo de estas perspectivas de las ciencias sociales en Trindade, coord. (2007)

${ }^{6}$ El volumen editado para un público fuera de América Latina por CLACSO (Bialakowsky, Gentilli et al 2012) da cuenta de algunas de las versiones en esta línea. 
Polis, Revista Latinoamericana, Volumen 14, $N^{\circ}$ 41, 2015

\section{Bibliografía}

Archer, Margareth (2009), Teoría social realista. El enfoque morfogenético. UAH, Santiago.

Bialakowsky, Alberto;Gentilli, Pablo eds. (2012), Latin American Critical Thought. Theory and CLACSO, Buenos Aires.

Comisión Gubelkian (1996), Abrir las ciencias sociales. Siglo XXI, México.

Faletto, Enzo y Fernando Henrique Cardoso (1968), Dependencia y desarrollo. Siglo XXI, México.

Garretón, Manuel Antonio (2000), La Sociedad en que vivi(re)mos. Introducción sociológica al cambio de siglo, LOM, Santiago.

Ídem (2007), “Ciencias sociales y sociedad en Chile: institucionalización, crisis y renacimiento”. En H. Trindade, coord, M.A. Garretón, G. De Sierra, M. Murmis y J.L. Reyna, Las Ciencias Sociales en América Latina en perspectiva comparada. Siglo XXI, México.

Ídem (2012), “La Problemática de América Latina y sus respuestas en juego”. En Mauro Salazar y Alejandro Osorio Rauld, editores, Democracia y Antagonismos en el Chile contemporáneo. Perspectivas posttransicionales.Editorial Akhilleus, Santiago, 2010

Hinkelammert, Franz (1984), Crítica de la razón utópica. Ed. Dei, San José de Costa Rica.

Medina Echavarría, José (1964), Consideraciones sociológicas sobre el desarrolloeconómico. Banda Oriental, Montevideo.

Mascareño, Aldo (2009), “Acción y estructura en América Latina. De la matrizsociopolítica a la diferenciación funcional”. En Persona y sociedad / UniversidadAlberto Hurtado Vol. XXIII / N² 2, Santiago.

Monsivais, Carlos (2001), Nuevo catecismo para indios remisos. LOM. Santiago.

Touraine, Alain (2013), La fin des sociétés. Editions du Seuil, Paris.

Trindade, Helgio, coord.; De Sierra, Gerónimo; Garretón, Manuel Antonio; Murmis, Miguel y Reyna, José Luis (2007), Las Ciencias Sociales en América Latina en perspectiva comparada. Siglo XXI, México. 
Stavenhagen, Rodolfo (2000), Conflictos étnicos y Estado nacional. Siglo XXI Editores, México.

Recibido: 10.08.2015

Aceptado: 25-08.2015 